\title{
Abdominal Aortic Aneurysm Repair in a Patient with Superior Mesenteric Artery Occlusion with Transient Intraoperative Infusion of the Inferior Mesenteric Artery: A Surgical Case Report
}

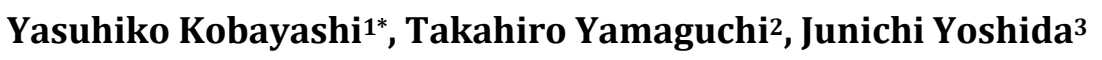 \\ ${ }^{1}$ Department of Cardiovascular Surgery, Amagasaki Central Hospital, Amagasaki, Japan \\ ${ }^{2}$ Department of Cardiovascular Surgery, Kawachi General Hospital, Osaka, Japan \\ ${ }^{3}$ Department of Cardiology, Amagasaki Central Hospital, Amagasaki, Japan \\ Email: *bw100kg@hotmail.co.jp
}

How to cite this paper: Kobayashi, Y., Yamaguchi, T. and Yoshida, J. (2018) Abdominal Aortic Aneurysm Repair in a Patient with Superior Mesenteric Artery Occlusion with Transient Intraoperative Infusion of the Inferior Mesenteric Artery: A Surgical Case Report. World Journal of Cardiovascular Surgery, 8, 213-218. https://doi.org/10.4236/wjcs.2018.811021

Received: September 26, 2018

Accepted: November 19, 2018

Published: November 22, 2018

Copyright ( $\odot 2018$ by authors and Scientific Research Publishing Inc. This work is licensed under the Creative Commons Attribution International License (CC BY 4.0).

http://creativecommons.org/licenses/by/4.0/ (c) (i) Open Access

\begin{abstract}
Abdominal aortic aneurysms (AAA) with mesenteric artery stenosis or obstructive lesions are occasionally recognized. While performing the graft replacement operation in such cases, if the collateral circulation supplying the gastrointestinal tract is well developed, it is necessary that this collateral circulation should be well perfused during aortic cross-clamping. A 72-year-old woman was admitted because of expansion of AAA. Computed tomography (CT) scan examination revealed occlusion at the origin of the superior mesenteric artery (SMA) and the development of a collateral circulatory pathway via the inferior mesenteric artery (IMA). During this operation, a vein cannula was placed in the IMA, through which blood was supplied with an extension tube for the protection of the intra-abdominal organs. The IMA was reimplanted after the graft replacement. Previous reports indicate that intestinal ischemia may be successfully prevented by supplying blood to the collateral circulation using various techniques. We report the surgical approach for treatment of AAA using a simple and convenient method to maintain intra-operative blood supply to vital organs.
\end{abstract}

\section{Keywords}

Abdominal Aortic Aneurysm, Occlusion of the Superior Mesenteric Artery, Transient Perfusion, Simple Method 


\section{Introduction}

Based on the previous reports, narrowing in the branches of the abdominal aorta ranges between $12.5 \%$ and $21 \%$ at the celiac artery (CA) and $3.4 \%$ and $37 \%$ at the SMA [1] [2]. In $25 \%$ of patients, stenosis of $50 \%$ or more is noted in the CA, in $6 \%$ of patients, in the SMA, and in $3.4 \%$ in both the CA and SMA [3]. Cases of patients having AAAs with associated stenosis or obstructive lesions in the CA, and SMA or IMA, due to atherosclerosis, have previously been reported. Rogers and colleagues [4] have reported cases of aortic reconstruction for obstructive lesions of the CA and SMA, and deaths due to lack of blood flow through the IMA after surgery. Sharafuddin and colleagues [5] have reported cases where stenting was performed to the CA and SMA during AAA repair, with subsequent development of significant collateral vessels. Colonic ischemia is a major complication of open and endovascular aneurysm repair (EVAR), with an incidence of $1 \%-6 \%$ [6]. When an AAA operation is performed on such patients, a wide range of ischemic injuries to the intra-abdominal organs might occur if ordinary aortic clamping is used [7]. Although the incidence of ischemic enteritis associated with the reconstruction of the abdominal aorta is $1.5 \%$ in the colon and $0.15 \%$ in the small intestine, the mortality rates are $40 \%$ and $90 \%$, respectively, which is considered a very poor prognosis [7]. Various methods have been employed to prevent ischemic injury to abdominal organs during surgery. In the following case, we report our experience using a simple technique, without requiring complicated operations such as bypass surgery, anastomosis, or extracorporeal circulation.

\section{Case Report}

A 73-year-old woman, previously diagnosed with AAA, was referred because expansion of AAA was detected on follow-up CTscan. She was $160 \mathrm{~cm}$ tall and weighed $52 \mathrm{~kg}$, with previous history of hypertension, hepatitis $\mathrm{C}$, and hyperlipidemia. She had no recent weight loss or symptoms of abdominal ischemia. Despite a daily dose of $1800 \mathrm{mg}$ of ethyl icosapentate, her hyperlipidemia was uncontrolled, and she had been a smoker for more than 45 years. Computed tomographic angiography (CTA) revealed occlusion (Figure 1(c)) in the SMA at its origin. Physical examination revealed a systolic murmur on abdominal auscultation, and the expanded IMA was confirmed radiologically as a long meandering extended vessel, which apparently was the collateral circulation that had developed (Figure 1(a)). The AAA was infra-renal type with a maximum short diameter of about $45 \mathrm{~mm}$ (Figure 1(b)). We decided to perform an EVAR after an angioplasty of the celiac trunk and the SMA. However, considering the significant calcification at the origin of the SMA and the associated risks, the family selected artificial blood vessel replacement surgery.

She was hospitalized to undergo surgery because the aneurysm had expanded by approximately $4 \mathrm{~mm}$ in 6 months.

Chest radiography was unremarkable, and cardiac catheterization revealed 


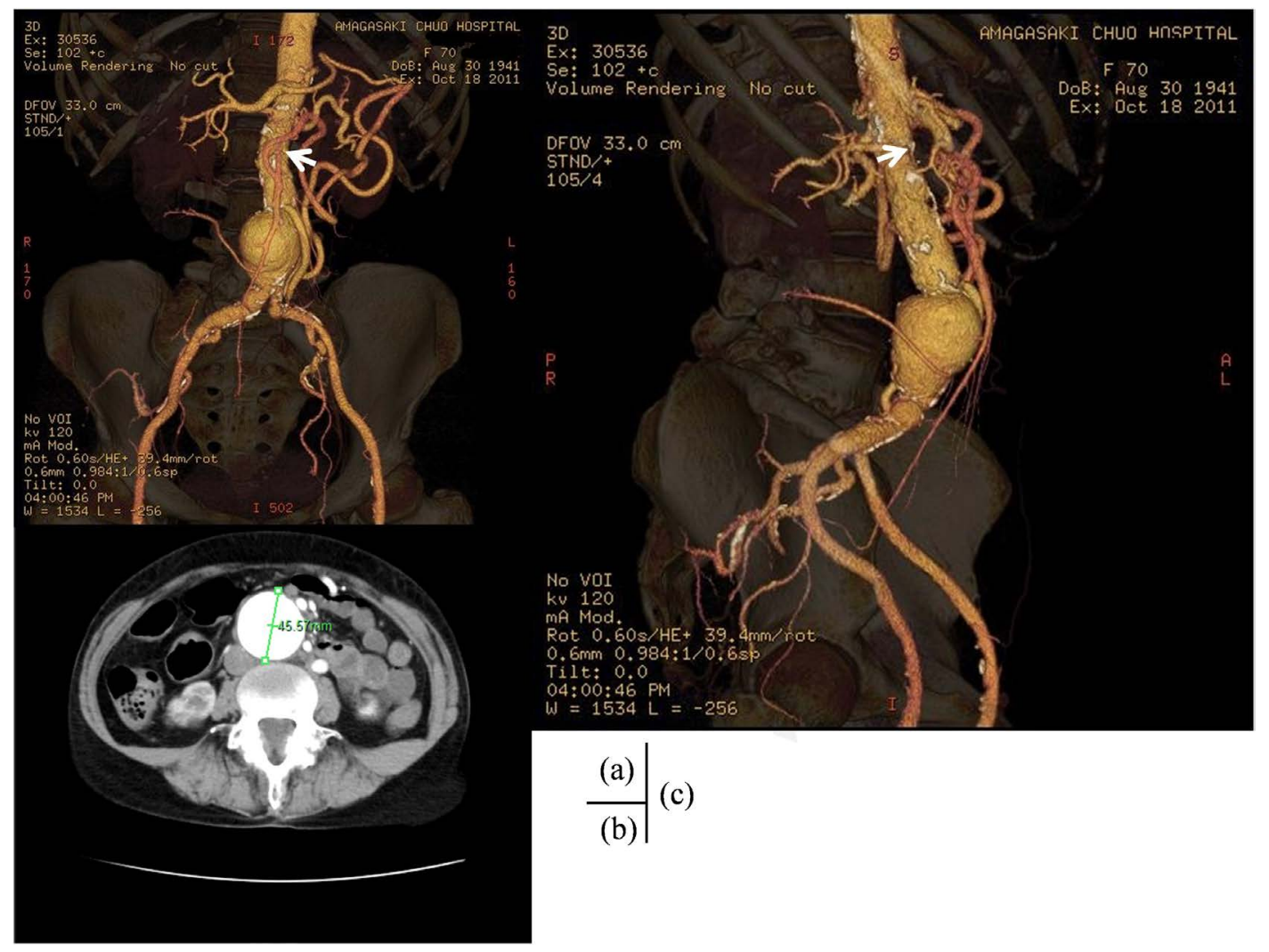

Figure 1. (a) Computed tomographic angiography (CTA) demonstrates an abdominal aortic aneurysm (AAA) by a three-dimensional (3D) image. The superior mesenteric artery (SMA) was occluded in the middle. Arrows point towards the SMA which was occluded in the middle. (b) The AAA was infra-renal type with a maximum short diameter of about $45 \mathrm{~mm}$. (c) The SMA was occluded at its origin. Arrows point towards the SMAs origin.

normal coronary arteries. In this patient, there was a high index of suspicion that if abdominal aortic clamping were to be performed in the infra-renal portion of the AAA in the usual manner, there would be a high risk of ischemic injury to the intra-abdominal organs. Therefore, the IMA supplying blood to the developed collateral blood vessels was perfused during the aortic reconstruction, to avoid ischemic injury to the intra-abdominal organs.

General and lumbar anesthesia were used for the operation, which was performed using a midline abdominal incision.

Laparotomy confirmed the presence of an AAA, and a thick IMA appeared meandering. The periphery of the IMA was appropriately peeled off and exposed to enable anastomosis, and the central side of the AAA was exposed and freed from surrounding tissues. After administration of heparin (100 units $/ \mathrm{kg}$ ), an 8-Fr sheath was placed centrally through the neck of the AAA with a vein cannula for the extension tube, and the vein cannula for coronary artery bypass grafting (CABG) was placed inside the vein cannula for the extension tube. The vein cannula for CABG was connected to the IMA and a syringe. We measured the maximum diameter of the vessel in the central part of the AAA to be approximately $18 \mathrm{~mm}$, and a Y-shaped knitted Dacron graft $(16 \times 8 \mathrm{~mm})$ manu- 
factured by MAQUET was chosen. After completion of the central anastomosis of the AAA, the IMA was anastomosed to the left leg of the Y-shaped knitted Dacron graft using 6-0 prolene RB-1. The vessel was unclamped, and blood flow to the IMA was resumed. Next, the left and right legs of the artificial blood vessel were sequentially anastomosed to the common iliac arteries (CIAs) with 6-0 Prolene. After sufficient de-airing of the artificial blood vessel, we unclamped and restored blood flow to the lower limbs. The procedure was completed after ensuring adequate hemostasis and closure. Duration of intestinal ischemia (time period between the clamping of the aorta and completion of the IMA anastomosis) was approximately 40 minutes. The patient recovered well postoperatively except for the development of systemic eczema that required a brief hospital stay. Postoperative CTA was performed (Figure 2), and she was discharged on the postoperative day 20 .

She was followed up to 25 months and no further adverse events noticed. CT scan and ultrasound examination showed regular results with a patent IMA branch.

\section{Discussion}

Patients developing ischemic colitis often follow a fatal course [7]. Several cases

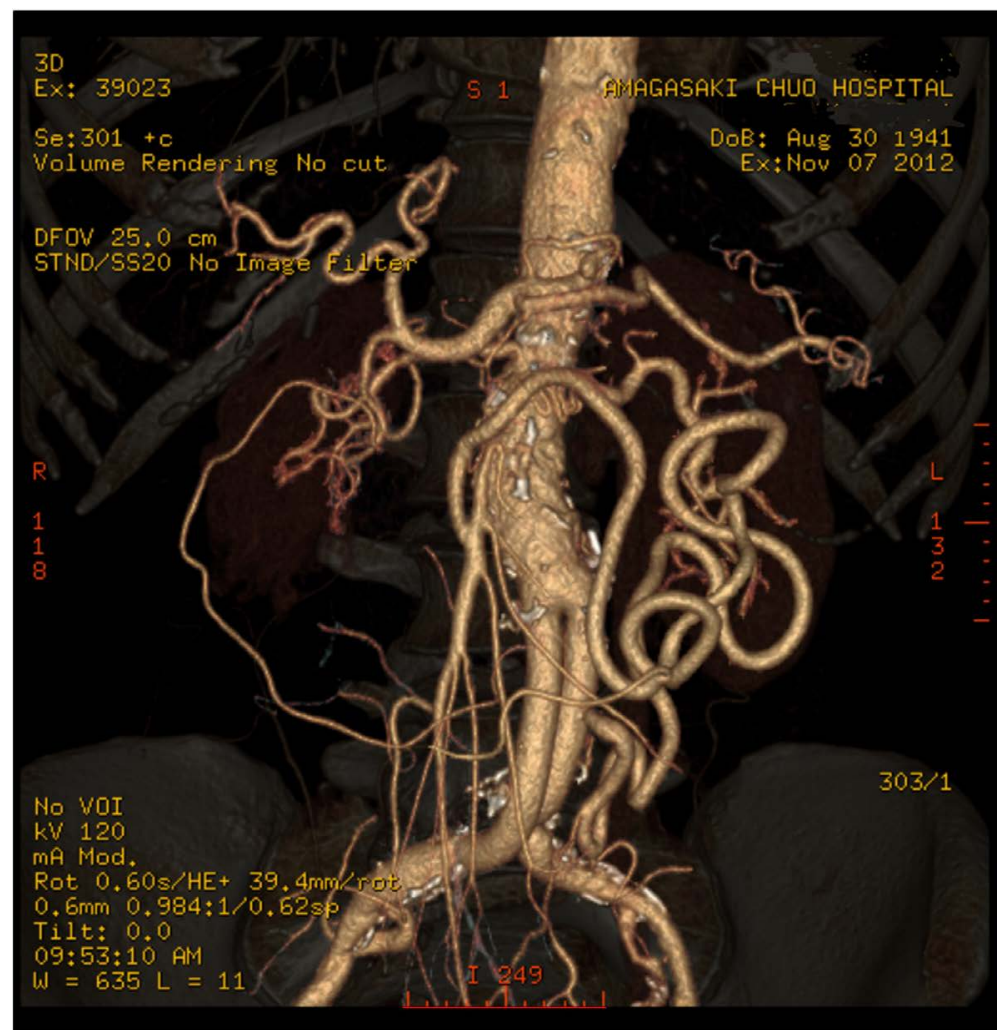

Figure 2. Postoperative CTA illustrating the relationship between the graft replacement (GR) and the IMA. A Y-shaped knitted Dacron graft $(16 \times 8 \mathrm{~mm})$ is chosen. After completion of the central anastomosis of the AAA, the IMA is anastomosed to the left leg of the Y-shaped knitted Dacron graft. 
have been reported wherein blood needed to be delivered to the collateral circulation to prevent ischemic injury to intra-abdominal organs [8] [9]. As a crinical condition of this case, as noted in our patient, it is proposed that the IMA developed as a collateral to ensure adequate blood flow to the intestinal tract in the presence of stenotic obstruction of the CA and SMA. Therefore, when treating AAA, maintaining uninterrupted blood flow to the intestinal tract is a priority. Our treatment strategy involved treating the AAA with simultaneous maintenance of adequate blood flow to the intestinal tract during the procedure. Thus, as a treatment choice, angioplasty was performed and EVAR or laparotomy was performed to perform artificial vessel vascular surgery, or AAA artificial vessel vascular surgery was performed while maintaining adequate blood flow to the intestinal tract during surgery.

Angioplasty was considered risky in the presence of occlusion of the SMA. Having understood the risk and benefits of the procedure, which were explained to her, the patient herself chose to undergo a laparotomy.

The neck of the AAA was noted to be relatively long, IMA of the aorta immediately under the kidney, which is collateral, is reimplanted as the first step, and the AAA operation was then performed. However, there was an arteriosclerotic lesion in the arterial wall, and we did not select it considering the possibility to skip debris by executing side clamp.

Neglen and colleagues [10] have reported a case in which the entire blood supply to the abdominal organs was maintained via collateral circulation from the IMA using extracorporeal circulation (ECC) during aortic clamping. Additionally, they reported temporary shunting with grafts and tubes replaced between the upper and lower portions of the aortic clamp site and arterial-arterial bypass using only the pump from the arms artery. However, ECC requires complicated instrumentation, and transient artificial blood vessel bypass using the radial artery or clavicle artery though relatively simple, is not an easy alternative to ensure sufficient blood flow to the intestinal tract. Thus, we did not select this artery at this time because exposure of the clavicle artery would require a detailed operation, and we intended this procedure to be a simple technique.

In this case, though the use of the femoral artery (FA) indwelling kit was simple, and convenient, a disadvantage was that if the 8-Fr sheath is used, it is difficult to maintain the rate of blood flow or sufficient flow pressure. We placed a syringe with a 20 - or 30-cc chip on a three-way tube as a stand-by option for pumping blood manually if the infusion to the IMA could not be maintained. We also considered blood infusion into the IMA using a pump for blood transfusion. However, we were able to maintain a good rate of blood flow infusion (about $180 \mathrm{~mL} / \mathrm{min}$ ) and adequate blood pressure throughout the operation, which could be attributed to the shorter length of the circuit compared to a bypass operation using artificial blood vessels.

\section{Conclusions}

This simple method could minimize the time required for an anastomosis using 
artificial blood vessels during an AAA repair procedure.

We propose that this technique of ensuring a transiently sufficient blood flow to the intestinal tract is a minimally invasive method that can be easily performed without involving an elaborate vascular anastomosis surgery. A simple procedure that prevents ischemia of abdominal organs during surgery, it seems to be a feasible option for cases requiring perfusion to the collateral circulation.

\section{Conflicts of Interest}

The authors declare no conflicts of interest regarding the publication of this paper.

\section{References}

[1] Derrick, J.R., Pollard, H.S. and Moore, R.M. (1959) The Pattern of Arteriosclerotic Narrowing of the Celiac and Superior Mesenteric Arteries. Annals of Surgery, 149, 684-689. https://doi.org/10.1097/00000658-195905000-00009

[2] Bron, K.M. and Redman, H.C. (1969) Splanchnic Artery Stenosis and Occlusion. Incidence; Arteriographic and Clinical Manifestations. Radiology, 92, 323-328. https://doi.org/10.1148/92.2.323

[3] Valentine, R.J., Martin, J.D. and Myers, S.I. (1991) Asymptomatic Celiac and Superior Mesenteric Artery Stenosis Are More Prevalent among Patients with Unsuspected Renal Artery Stenosis. Journal of Vascular Surgery, 14, 195-199. https://doi.org/10.1067/mva.1991.29423

[4] Rogers, D.M., Thompson, J.E. and Garrett, W.V. (1982) Mesenteric Vascular Problems: A 26-Year Experience. Annals of Surgery, 195, 554-565. https://doi.org/10.1097/00000658-198205000-00004

[5] Sharafuddin, M.J., Olson, C.H. and Sun, S. (2003) Endovascular Treatment of Celiac and Mesenteric Arteries Stenosis: Applications and Results. Journal of Vascular Surgery, 38, 692-698. https://doi.org/10.1016/S0741-5214(03)01030-9

[6] Becquemin, J.P., Majewski, M. and Fermani, N. (2008) Colon Ischemia Following Abdominal Aortic Aneurysm Repair in the Era of Endovascular Abdominal Aortic Repair. Journal of Vascular Surgery, 47, 258-263. https://doi.org/10.1016/j.jvs.2007.10.001

[7] Johnson, W.C. and Nabseth, D.C. (1974) Visceral Infarction Following Aortic Surgery. Annals of Surgery, 180, 312-318. https://doi.org/10.1097/00000658-197409000-00010

[8] Fisher Jr., D.F. and Fry, W.J. (1987) Collateralmesenteric Circulation. Surgery, Gynecology, and Obstetrics, 164, 487-492.

[9] Ernst, C.B. (1983) Prevention of Intestinal Ischemia Following Abdominal Aortic Reconstruction. Surgery, 93, 102-106.

[10] Neglen, P., Eklof, B. and Shuhaiber, H. (1987) Temporary Extracorporeal Perfusion of the Splanchnic Circulation Dependent on the Riolan Anastomosis. The Journal of Cardiovascular Surgery, 28, 249-252. 